\title{
Ureteral Marginal Zone Lymphoma of Mucosa-Associated Lymphoid Tissue, Chronic Inflammation, and Renal Artery Atherosclerosis
}

\author{
Hojung Lee · Jong Eun Joo · Young Ok Hong · Won Mi Lee \\ Eun Kyung Kim · Jeong Joo Woo ${ }^{1}$ Soo Jung Gong ${ }^{2}$ · Jooryung Huh ${ }^{3}$ \\ Departments of Pathology, ${ }^{1}$ Radiology, and ${ }^{2}$ Internal Medicine, Eulji General Hospital, Eulji University College of Medicine, Seoul; \\ ${ }^{3}$ Department of Pathology, Asan Medical Center, University of Ulsan College of Medicine, Seoul, Korea
}

Malignant lymphoma of the upper urinary tract including the renal pelvis and ureter is extremely rare. Less than ten cases have been reported in the literature, of which extranodal marginal zone lymphoma of mucosa-associated lymphoid tissue (MALT lymphoma) is the most frequent histologic type. ${ }^{1}$ MALT lymphoma arising in the stomach, skin, thyroid, and salivary gland is associated with chronic inflammation caused by pathogenic microorganisms or autoimmune disorder; ${ }^{2}$ however, the pathogenesis of MALT lymphoma in the upper urinary tract is unknown. Herein, we report a case of upper ureteral MALT lymphoma with extensive peri-ureteropelvic inflammation and atherosclerosis of the renal artery, and discuss the possible correlation between these lesions.

\section{CASE REPORT}

A 73-year-old male patient was admitted to the emergency room with a chief complaint of right flank pain for one week. Laboratory tests showed an elevated white blood cell count of $13.21 \times 10^{3} / \mu \mathrm{L}$ (neutrophilic leukocytes, $85 \%$; lymphocytes, $5 \%$ ) and high C-reactive protein (CRP) level of $23.71 \mathrm{mg} / \mathrm{dL}$. Urine analysis was unremarkable, and no microbial growth was observed in blood or urine cultures. Abdominal computed tomography (CT) showed diffuse thickening of the right uretero-

Corresponding Author Jooryung Huh, MD, PhD

Department of Pathology, Asan Medical Center, University of Ulsan College of

Medicine, 88 Olympic-ro 43-gil, Songpa-gu, Seoul 138-736, Korea

Tel: +82-2-3010-4553, Fax: +82-2-472-7898, E-mail: jrhuh@amc.seoul.kr

Received: March 24, 2015 Revised: April 24, 2015

Accepted: April 27, 2015 pelvic wall with hydroureteronephrosis (Fig. 1A). Urine cytology and washing cytology of the right ureter revealed no atypical cells. Right nephroureterectomy was performed for histologic diagnosis, and the intraoperative diagnosis of lymphoid malignancy was made. Positron emission tomography/CT revealed multiple hypermetabolic lesions throughout the neck, chest, and abdomen, suggesting lymphoma seeding (Fig. 1B). The resected specimen showed an elongated, concentric, whitish yellow solid lesion along the walls of the renal pelvis and proximal ureter (Fig. 2). This lesion also surrounded the atherosclerotic renal artery (Fig. 2, upper inset).

Histologic examination revealed peri-ureteropelvic nodular lymphoid infiltrates encroaching upon the ureter wall (Fig. 3A). The perimuscular layer of the ureter was heavily infiltrated with tumor cells. There were centrocyte-like cells with slightly irregular nuclear contours and clear cytoplasm, occasional larger activated cells, monocytoid cells, and plasma cells forming numerous lymphoid follicles. Immunohistochemically, the neoplastic cells were positive for CD20 (Fig. 3B) but negative for CD3, CD5, CD10, Bcl-2, Bcl-6, and cyclin D1. Tumor-infiltrating T cells were mostly CD4+ cells (Fig. 3C). Monotypic expression of $\lambda$ light chain was demonstrated in the plasma cells. This case was diagnosed as a MALT lymphoma. Adjacent peripelvic adipose tissue showed a dense inflammatory infiltrate composed of lymphoplasma cells, eosinophils, and histiocytes with fibroblastic proliferation (Fig. 3D). Infiltrating lymphocytes were mainly CD4+ T cells, and CD20 + B cells were rare. Neither IgG4-positive plasma cells nor anaplastic lymphoma kinase-stained cells were noted, excluding the possibility of IgG4-associated disease or an inflammatory myofibroblastic tumor. 

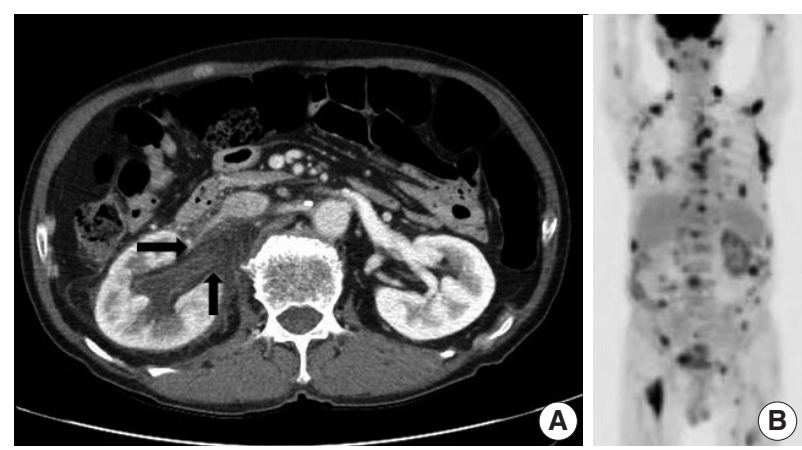

Fig. 1. (A) Contrast-enhanced axial computed tomography scan of the abdomen shows thickening with enhancement of the right renal pelvis wall with perinephric soft tissue infiltration (arrows) and hydroureteronephrosis. (B) Positron emission tomography computed tomography reveals hypermetabolic lesions in multiple neck, axillary, mediastinal, and pelvic lymph nodes as well as the chest, abdominal wall, left parotid gland, and right thigh.

The patient received rituximab-cyclophosphamide, doxorubicin, vincristine, and prednisone (R-CHOP) chemoimmunotherapy. Complete remission was achieved with no recurrence at the 14-month follow-up.

\section{DISCUSSION}

Lymphomas constitute approximately $5 \%$ of non-urothelial tumors of the urinary tract, affecting urinary bladder in more than $90 \%$ of cases. ${ }^{3}$ In contrast, lymphoma of the upper urinary tract is extremely rare. Of the reported cases, MALT lymphoma is the most frequent, with eight case reports; ${ }^{1,4-7}$ seven in the renal pelvis and one in the upper ureter. While MALT lymphoma of the urinary bladder is frequently associated with chronic cystitis and female predominance, ${ }^{3,8}$ MALT lymphoma of the upper urinary tract typically affects middle-aged or elderly males with unknown preceding conditions. ${ }^{1}$ MALT lymphoma of the urinary tract presents with incidental mass or thickening on radiographs, although some may complain of pain. ${ }^{1,4-7}$ It usually presents at a localized stage and has excellent prognosis, although dissemination can occur, as in the present case. ${ }^{1,9}$ Despite its widespread extent, the primary origin in the urinary system of this case was supported by the growth pattern within the renal pelvis and ureter walls. However, the possibility of secondary ureteral involvement of MALT lymphoma in other organs still remains since histologic confirmation at other sites was not performed. Unlike its gastric or even bladder counterpart, ureteropelvic MALT lymphoma rarely shows subepithelial lymphoid infiltrates or lymphoepithelial lesions, mainly involving periureteral fat. ${ }^{1,4-8}$ Thus, ureteral MALT lymphoma is probably not as-

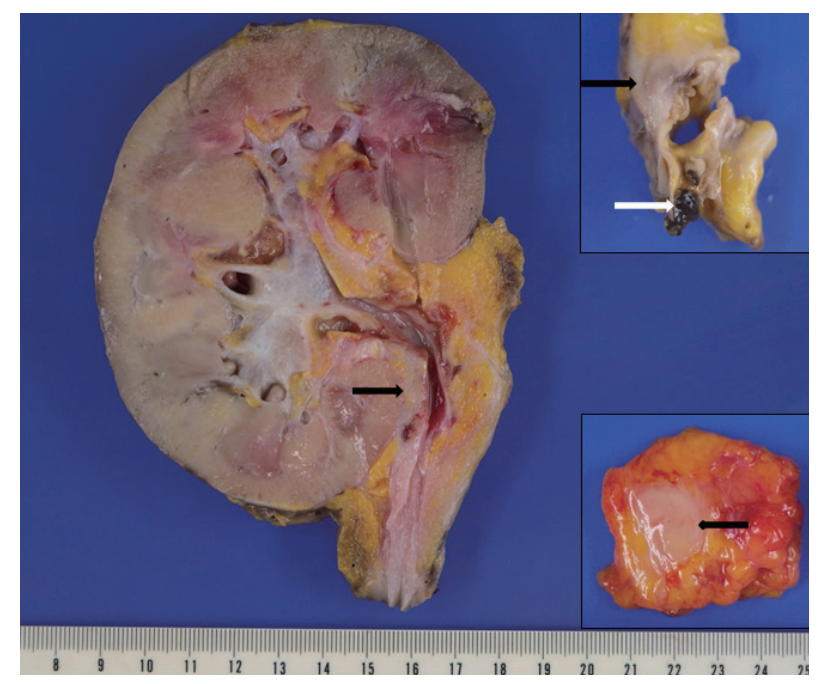

Fig. 2. Grossly, the specimen shows whitish yellow solid lesions along the renal pelvis and the ureter (black arrow), forming a concentric mass (black arrow, lower inset) compressing the ureter lumen. Renal artery surrounded by white solid lesions (black arrow, upper inset) shows atheromatous plaques and a thrombus (white arrow, upper inset).

sociated with 'mucosa' or luminal insults but rather with periureteral inflammation. Therefore, in the case of lymphoma accompanied by inflammatory lesion, it may be better to be called 'inflammation-associated lymphoid tissue lymphoma' rather than MALT lymphoma, if MALT is not present, as in this case.

In MALT lymphoma, a chronic inflammatory microenvironment is important for the development of the tumor. ${ }^{2}$ The intratumoral T-cell component is a crucial factor, and $\mathrm{CD} 4+\mathrm{T}$ cells activate $\mathrm{B}$ cells in a CD40-dependent manner in combination with Th2 cytokines (interleukin [IL] 4 and/or IL-10). ${ }^{2}$ Thus, the chronic inflammatory infiltrates seen in the peripelvic area in the present case may have provided the appropriate conditions for growth of a malignant clone.

On the other hand, atherosclerosis is defined as a chronic inflammatory response of the arterial wall to endothelial injury triggered by a variety of insults including lipid accumulation. ${ }^{10}$ Many inflammatory cells, mostly monocytes and CD4 $+\mathrm{T}$ cells, are recruited into the arterial intima, forming atheromatous plaques and accompanied by high blood levels of acute phase reactants such as CRP, as in the present case. ${ }^{10}$ Renal artery atherosclerosis in our patient may be a coincidental lesion considering his old age. However, the patient's laboratory and pathologic findings indicated that the inflammatory process was concurrently ongoing within the renal artery and peripelvic adipose tissue. Therefore, in the present case, the final outcome may 

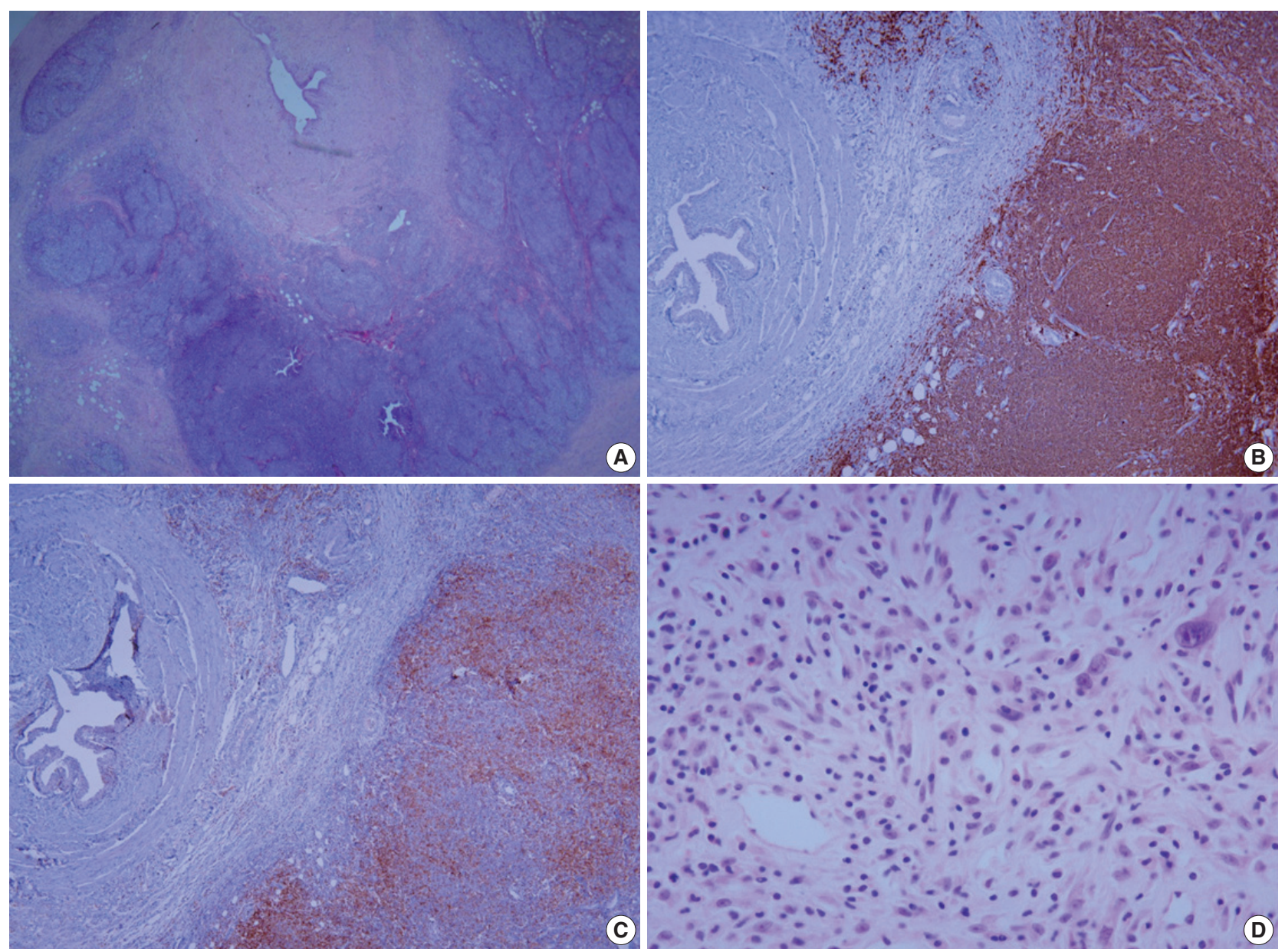

Fig. 3. Microscopic features of mucosa-associated lymphoid tissue lymphoma and adjacent chronic inflammation. (A) Tumor cells infiltrating the perimuscular layer of the ureter form lymphoid follicles with follicular colonization. Immunohistochemically, tumor cells are diffusely positive for CD20 (B) and accompanying T cells are mostly CD4 positive (C). (D) Peripelvic adipose tissue shows a mixed infiltrate of lymphoplasma cells, eosinophils, and histiocytes with fibroblastic proliferation.

manifest as ureteral MALT lymphoma and renal artery atherosclerosis.

\section{Conflicts of Interest}

No potential conflict of interest relevant to this article was reported.

\section{REFERENCES}

1. Otsuki H, Ito K, Sato K, et al. Malignant lymphoma of mucosa-associated lymphoid tissue involving the renal pelvis and the entire ureter: a case report. Oncol Lett 2013; 5: 1625-8.

2. Thieblemont C, Bertoni F, Copie-Bergman C, Ferreri AJ, Ponzoni M. Chronic inflammation and extra-nodal marginal-zone lymphomas of MALT-type. Semin Cancer Biol 2014; 24: 33-42.

3. Eble JN, Sauter G, Epstein JI, Sesterhenn IA. World Health Organi- zation classification of tumours: pathology and genetics tumors of the urinary system and male genital organs. Lyon: IARC Press, 2004.

4. Araki K, Kubota Y, lijima Y, et al. Indolent behaviour of low-grade B-cell lymphoma of mucosa-associated lymphoid tissue involved in salivary glands, renal sinus and prostate. Scand J Urol Nephrol 1998; 32: 234-6.

5. Qiu L, Unger PD, Dillon RW, Strauchen JA. Low-grade mucosa-associated lymphoid tissue lymphoma involving the kidney: report of 3 cases and review of the literature. Arch Pathol Lab Med 2006; 130: 86-9.

6. Mita K, Ohnishi Y, Edahiro T, Fujii T, Yamasaki A, Shimamoto F. Primary mucosa-associated lymphoid tissue lymphoma in the renal pelvis. Urol Int 2002; 69: 241-3.

7. Hara M, Satake M, Ogino H, et al. Primary ureteral mucosa-associated lymphoid tissue (MALT) lymphoma: pathological and radio- 
logical findings. Radiat Med 2002; 20: 41-4.

8. Matsuda I, Zozumi M, Tsuchida YA, et al. Primary extranodal marginal zone lymphoma of mucosa-associated lymphoid tissue type with malakoplakia in the urinary bladder: a case report. Int J Clin Exp Pathol 2014; 7: 5280-4.
9. Thieblemont C, Berger F, Dumontet C, et al. Mucosa-associated lymphoid tissue lymphoma is a disseminated disease in one third of 158 patients analyzed. Blood 2000; 95: 802-6.

10. Hansson GK, Robertson AK, Söderberg-Nauclér C. Inflammation and atherosclerosis. Annu Rev Pathol 2006; 1: 297-329. 